\title{
Study On the Key Points Analysis and Training Strategies of Tennis Intercept Technique
}

\author{
Yuan Zhang \\ Northwest University, Xi’an, China, 710069
}

Keywords: tennis intercept technique; technical points; training strategy

\begin{abstract}
The sports cause in our country continues to develop and the concept of national fitness is deeply rooted. In a number of sports, tennis has its own particularities. There are many technical points in tennis. Athletes who want to improve their professional skills must adopt effective training strategies. Tennis intercept technique is the most important thing in tennis practice. Only by mastering the technique of tennis intercept can the athlete break the opponent's defense and win the match. From this perspective, athletes should carry out tennis intercept practice in their daily training. This paper specifically discusses the technical points and training strategies of tennis intercept to provide some references for related parties.
\end{abstract}

\section{Introduction}

In the analysis of tennis, we can find that tennis intercept technology is crucial. Tennis intercept technology is also called blocking, and in the process of the game, the racket should be used to intercept the ball and let the tennis get half volley with push. Tennis returns faster, and most opponents can't react to the returned tennis ball in time. Athletes can get points from it. For now, tennis intercept technology has become one of the necessary technologies for offensive players. Many excellent athletes have used tennis intercept technology to win the game. With the continuous development of the sports cause, the application range of tennis intercept technology has become more and more extensive. Many athletes have learned this technology and improved their ability to hit the ball. From this point of view, tennis intercept technology has a prominent beneficial effect on athletes. Athletes should analyze the technical points of tennis intercept and adopt efficient training methods.

\section{Technical Points of Tennis Intercept Technique}

\subsection{Racket grasp technology}

In the process of learning tennis intercept technology, athletes must first master the scientific racket grasp method. In the intercept of opponent's ball, the athlete can adopt the continental grasp method. Compared with other grasp methods, the continental grasp method is more flexible. Whether it is a forehand grasp or a backhand grasp, a good intercept effect can be obtained ${ }^{[1]}$. Even if the opponent's ball is moving faster, the athlete can react quickly and make an intercept closing to the net. When the two sides confront each other before the net, the intercept is more difficult. Athletes do not have time to change the racket grasp method, and there is no way to accurately determine the direction of tennis. Watching a game of tennis, we can find that many athletes lose points because of the miscalculation of tennis ball direction. Therefore, athletes must be prepared to intercept the other's tennis ball in advance. In order to avoid wasting time by changing the grasp method, the athlete may need to adopt a continental grasp method and perform a sensitive reaction when the opponent hits the ball.

\subsection{Adjust the posture}

In the intercept, the athlete's posture is very important. In general, the center of gravity of the athlete's body should be slightly forward, and the athlete's heel should not completely fit the ground. 
They should maintain a certain distance. The athlete's knees should move closer to the inside, thus the body's direction can be quickly adjusted, which improves the accuracy of the tennis intercept. Many athletes are physically upright and back straight during the intercept, which has a very negative impact on the intercept. Due to the stiffer body, the athlete's reaction to tennis ball is greatly delayed. In addition to paying attention to the posture of back, foot and leg, hand posture is equally important. The two hands of the athlete should be in a state of forward stretching, and the racket should be diagonally above the head ${ }^{[2]}$. If the racket is too close to the athlete's head, his two hands are too correct and it will also affect the quality of the tennis intercept.

\subsection{Judge the tennis ball accurately}

As mentioned above, the direction of tennis ball is not easy to judge, so athletes should watch it carefully and hit the ball in front of the body. Many athletes are struggling to achieve the goal of hitting the ball in front of the body. It's caused by several reasons: firstly, some athletes are not prepared enough to accurately determine the direction of the ball. Secondly, some athletes hit the ball too far behind and they could not handle the ball. Both of the above reasons have a direct impact on the efficiency of tennis intercept and it is easy for athletes to lose their points. In order to avoid the problems above, athletes should do as the following: firstly, athletes should prepare in advance, put the racket straight, and predict the direction of the tennis ball. Thirdly, the athlete should ensure that the hitting point is moderate to easily intercept and control the ball.

\subsection{Horizontal backswing}

When intercepting a tennis ball, the athlete should adjust his/her own control of the racket so as to avoid excessive swings. Many athletes are nervous on the field and unconsciously increase their swing effort when they see the tennis ball, making tennis intercept missed. In fact, tension can cause the hand to fall into a state of excessive excitement. Athletes should adjust their mentality, estimate the speed of the opponent's ball, and adjust the intensity of racket grasp. The angle of the backward backswing cannot be too great. If the angle is too great, the difficulty of catching the ball will increase significantly. In addition to controlling the strength, the athlete should also ensure the horizontal backswing is taken so that the racket reacts to the tennis ball on the horizontal cutting line in the direction of tennis ball.

\subsection{Control the angle}

The wrist is the key of the racket grasp. Generally speaking, keeping the wrist and racket in a vertical position is the best times for tennis interact. After the angle is formed, the upright state of racket can be maintained, and the tennis ball can be actively intercepted to reduce the error rate of the hitting posture. If the wrist and racket do not remain vertical, it is difficult for the athlete to grasp the trend of the tennis ball and control it accurately. Athletes generally have a problem in the field: the faster the opponents serve, the greater strength of the athlete's racket grasp; the slower the opponents serve, the weaker strength of the athlete's racket grasp. In fact, the speed of serving is not related to the strength of racket grasp. Athletes should not be affected by the opponent's serve but should focus on their wrists. From the initial stage of the game, the athlete should adjust the angle of the wrist and racket. When the racket position is changed, the position of the wrist should also be changed.

\section{Training Strategies of Tennis Intercept Technique}

\subsection{Standard practice method}

In the initial stages of learning tennis intercept technology, athletes should use standard practice methods. Athletes should imagine that tennis is alive and be familiar with the characteristics of tennis ${ }^{[3]}$. The methods of forehand practice and backhand practice show certain differences. Athletes should be trained in groups to grasp different training methods. Take forehand practice as an example. Athletes should use their left hands in front of the net to catch the right-hand ball hit by the opponent. In the process of catching the ball, the athlete should keep striding forward and 
quickly grab the tennis ball, then move the tennis ball to the front of the neck and block the ball with the throat of the racket. After long-term training, athletes can learn more about the characteristics of tennis. After the forehand practice are completed, the athlete can change intercept posture, use the middle of the handle to block the ball, and then use the bottom to block ball.

Tennis intercept technology has the characteristics of precision. Athletes should ensure the standardization of training. In order to check whether the movement is up to standard, the athlete can practice in the mirror, repeatedly making forehand practice and backhand practice, and examining the normalization of forehand and backhand practice. In order to continuously improve the difficulty of exercise, the athlete can place an obstacle behind it to minimize the movement. Athletes who have just contacted the technique of tennis intercept can stand before the wall to carry out training. If the racket hits the wall, it means that the player's swing is too strong. Many athletes take a swing too great and cause damage to the racket, but the hitting power must be ensured moderate for tennis intercept technology.

\subsection{Practice methods for advancement}

After learning the standard practice method, athletes can use the method of advancement practice. Advancement practice methods need to be divided into two groups. The athletes must cooperate with a partner to complete intercept practice. The two sides in the practice should maintain a space of about two meters. One athlete should bounce the ball for five times with the racket, and then pass the tennis ball to another athlete, and let the other athlete bounce the ball for five times. After repeated ten rounds, the number of bouncing can be reduced, from five to four, from four to three, etc. ${ }^{[4]}$. After a long period of independent practice, the athlete can properly pull himself apart from another athlete. In general, advancement practice methods begin with forehand practice and then extend to backhand practice.

After the basic practice is completed, two athletes can perform intercept practice in front of the net. The distance between the two athletes should be about four meters away from the net, and they should stand along the slash net, alternating forehand practice and backhand practice. After the coach has served the ball, the athlete will intercept the tennis ball according to the established practice goal and reach the designated position.

\subsection{High level practice method}

Most sports training methods are progressive and tennis intercept technology has no exception. After many preliminary trainings and advancement trainings, athletes can use high level practice. There are many modes of high level practice methods: Firstly, athletes can use two-person practice. The coach serves at the baseline and the athlete intercepts the tennis ball and quickly moves his or her footsteps, touches the marker, and then moves to the designated area to intercept the ball again. Secondly, athletes can use three-person practice ${ }^{[5]}$. An athlete is at the bottom line. An athlete is in front of the net. Two athletes a group plays with another athlete to carry out tennis intercept practice in turns. Again, athletes can use a two-on-two four-person practice. In the application of the four-person practice method, each athlete has a fixed position, and athletes who play straight balls and slash balls should be exchanged at any time. Finally, athletes can use the practice of four people intercept. Two people stand in front of the net, two people standing at the position of bottom line, alternating tennis intercept training.

\section{Conclusion}

To sum up, China's sports cause has continued to develop and tennis has achieved a series of achievements. There are many technologies in tennis that are crucial to athletes. In several tennis technologies, intercept technology occupies a very important position. Many athletes have mastered the main points of tennis intercept technology and won in the competition. The mastery of the tennis intercept does not happen in an action. Athletes should analyze the technical points of tennis intercept technologies and adopt efficient tennis intercept training methods. 


\section{References}

[1] Fengling Li, Kelei Li, Jingjing Lv. Research on Biomechanics Technology Based on the Tennis Sports. Springer London:2013-06-15.

[2] Linghang Yang. Application of the Mechanical Impedance of Body Arm Movement Based on the Virtual Tennis System. Advanced Materials Research,2013,2649(791).

[3] Robert Laudone, Eric W. Liguori, Jeffrey Muldoon, Josh Bendickson. Technology brokering in action: revolutionizing the skiing and tennis industries. Journal of Management History,2015,21(1).

[4] Zhang Songkui, Li Yilong. Analysis of the Characteristics on the Use of Skills and Tactics in the Chinese Men's Soft Tennis Doubles Game. Journal of Nanjing Institute of Physical Education (Natural Science), 2015, 14(01): 56-60.

[5] Liao Yubing, Liao Xiaobin, Zhou Meifang, Zheng Guohua, Liu Chuang. Research on the technical methods to win the world's best men's and women's single competition of tennis - taking 2010 “ATP singles finals" and "WTA singles year-end finals” as an example. China Sport Science and Technology,2012,48(06):35-40+47. 